\title{
On the need of creating multicenter registries for more suitable matched cohort studies to elucidate best multidisciplinary care strategies for patients with Chiari malformations
}

\author{
Ekkehard M. Kasper ${ }^{1}$ (D) \\ Received: 14 December 2020 / Accepted: 16 December 2020 / Published online: 2 February 2021 \\ (C) Springer-Verlag GmbH Austria, part of Springer Nature 2021, corrected publication 2021
}

Dear Editor,

We greatly appreciate the comments made by Dr. Roper and her team of co-authors and their corroborative statement to seek multidisciplinary evaluation for optimal peripartum management of pregnant women with a Chiari I malformation. Their experience and our own data are suitable to question the frequently encountered recommendation to pursue an elective $\mathrm{C}$-section in this patient population.

Dr. Roper and her team had reported their own experience from the UK in a manuscript on the topic in 2018, which observed similar outcomes in their own institutional study cohort of patients harboring essentially asymptomatic Chiari I malformations. Their study period from a large volume tertiary maternity unit in the UK stretched a 14-year period during which 21 patients such were successfully managed. Twothirds $(15 / 23)$ of the deliveries in their cohort occurred as normal vaginal deliveries, with only eight being induced for obstetric indications, with the remaining deliveries occurring by $\mathrm{C}$-section. All pregnancies were carried to term, and none were associated with any adverse neurological outcomes at discharge. This reflects excellent interdisciplinary antepartum and intrapartum management in our opinion, and the result of a healthy outcome for both mother and child is not diminished by the potential of selection bias due to the observational design as the authors consider themselves.

Given that our systematic PRISMA-guided literature search covered the MRI era after 1991 until the beginning of 2018 , this part of our study was conducted just prior to the publication date of the work she and her team had embarked on. Due to some changes in the format of our own paper from a case series to a comprehensive review of the existing body of the literature, we faced some delays in generating the final version of our manuscript, and we regret that her paper was not retrieved at a later point in time for inclusion in the discussion section, as it is a valuable addition to the literature on the topic. Taken together with our own review and extensive discussion on the matter, both our author team and Dr. Roper and her colleagues hope that other providers will be motivated to create multicenter registries for more extensive matched cohort studies to elucidate best care strategies in this vulnerable patient population.

Publisher's note Springer Nature remains neutral with regard to jurisdictional claims in published maps and institutional affiliations.

This article is part of the Topical Collection on Brain Tumors

Ekkehard M. Kasper

kaspere@mcmaster.ca

1 McMaster University Faculty of Health Sciences, Service Head, Neurosurgery, Hamilton General Hospital, 237, Barton Street East, McMaster Clinic Wing, Rm 728, Hamilton, ON L8L 2X2, Canada 